\title{
医用放大镜辅助口腔黏膜病临床视诊的价值
}

张羽婷, 袁培养, 江涵, 仇学梅, 王冏珂, 罗小波, 但红霞, 周 瑜, 曾 昕, 江潞, 陈谦明*

四川大学华西口腔医院口腔黏膜病科口腔疾病研究国家重点实验室国家口腔疾病 临床医学研究中心,四川成都 610041

[摘 要] 目的: 研究医用放大镜在提高口腔慗膜病临床诊断效率中的作用及其 最适合的临床使用参数。方法: 选取 24 例斑块型口腔扁平苔藓及均质型口腔白斑 病病例, 以组织病理学检查结果为标准, 评价经肉眼视诊和医用放大镜辅助视诊的 敏感度、特异度和准确度; 以问卷调查方式研究医用放大镜辅助视诊对口腔慗膜 病诊断的主观有效率、使用体验并探讨其最适合的临床使用参数。结果: 医用放 大镜辅助视诊鉴别斑块型口腔扁平苔藓及均质型口腔白斑病的敏感度、特异度、 准确度分别为 $94.74 \% 、 100.00 \% 、 95.83 \%$, 均高于肉眼视诊的敏感度、特异度、 准确度 $(89.47 \% 、 80.00 \% 、 87.50 \%)$ 。医生使用的主观评价认为医用放大镜辅助 诊断的有效率为 $91.76 \%$ 。腔踳膜病临床诊治最适参数为放大倍数 3.5 倍, 工作 距离 $400 \mathrm{~mm}$ 。结论: 医用放大镜能够有效提高口腔恭膜病的视诊诊断效率, 且具有 方便、实时的特点。推荐临床使用参数为放大倍数 3.5 倍, 工作距离 $400 \mathrm{~mm}$ 。

[关键词] 医用放大镜; 口腔秥膜病; 斑块型口腔扁平苔藓; 均质型口腔白斑病; 辅助 诊断; 使用体验

[中图分类号 ］R781.5 [ 文献标志码 ］A

\section{Application of medical magnifying loupes in diagnosis of oral mucosal diseases}

ZHANG Yuting, YUAN Peiyang, JIANG Han, QIU Xuemei, WANG Jiongke, LUO

Xiaobo, DAN Hongxia, ZHOU Yu, ZENG Xin, JIANG Lu, CHEN Qianming (Department of Oral Medicine, West China Hospital of Stomatology, Sichuan University, State Key Laboratory of Oral Diseases, National Clinical Research Center for Oral Diseases, Chengdu 610041, China)

Corresponding author: CHEN Qianmin, E-mail: qmchen@zju.edu.cn, https://orcid.org/

收稿日期: 2021-01-25接受日期:2021-04-21

基金项目:国家自然科学基金(81730030)

第一作者:张羽婷, 住院医师, 主要从事口腔黏膜病的相关研究; E-mail : 1501379093@qq.com; https ://orcid.org/0000-00018009-0840

通信作者:陈谦明,教授,主任医师,博士生导师,主要从事口腔黏膜病的病因及防治研究;E-mail :qmchen@zju.edu.cn; https ://orcid.org/0000-0002-5371-4432

*现在浙江大学医学院附属口腔医院 浙江大学口腔医学院 浙江省口腔疾病临床医学研究中心 浙江省口腔生物医学重点 实验室 浙江大学癌症研究院工作 
0000-0002-5371-4432

[ Abstract ] Objective: To evaluate the application of medical magnifying loupes in diagnosis of oral mucosal diseases. Methods: Twenty-four patients with plaque-type oral lichen planus or homogeneous oral leukoplakia were inspected by naked eyes or assistance with magnifying loupes. Histopathological results were used as the gold standard to evaluate the sensitivity, specificity and accuracy of the two methods in clinical diagnosis. Questionnaires were used to evaluate the subjective effect of magnifying loupes on the diagnosis efficiency of oral mucosal diseases and to explore the most suitable parameters for application. Results: The sensitivity, specificity and accuracy of medical magnifying loupes for the identification of plaque-type oral lichen planus and homogeneous oral leukoplakia were $94.74 \%, 100.00 \%$ and $95.83 \%$, respectively, which were significantly higher than those of naked eye inspection $(89.47 \%, 80.00 \%$ and $87.50 \%$ ). The effective rate of magnifying loupes assisted diagnosis was $91.76 \%$ according to physicians' subjective evaluation. The most suitable parameters were 3.5 times magnification and $400 \mathrm{~mm}$ working distance. Conclusion: The medical magnifying loupes can effectively improve the efficiency of the inspection and diagnosis of oral mucosal diseases, and have the characteristics of convenience and real-time. The recommended clinical parameters are 3.5 times magnification and $400 \mathrm{~mm}$ working distance.

[ Key words ] Medical magnifying loupe; Oral mucosal diseases; Plaque-type oral lichen planus; Homogeneous oral leukoplakia; Auxiliary diagnosis; User experience

[J Zhejiang Univ (Med Sci), 2021, 50(2): 205-211.]

口腔黏膜病是指主要累及口腔黏膜组织及软 组织的各类疾病, 病损的重叠性是其重要的临床 特征,也是诊断的难点 ${ }^{[1]}$ 。准确收集病损信息是 口腔黏膜疾病诊断的重要依据。在基于肉眼的临 床检查中, 某些微小隐匿性病损或某些病损的细 微结构往往被忽视, 使得准确诊断变得困难。医 用放大镜是一种非侵人性视觉放大技术 ${ }^{[2]}$, 可用 于观察黏膜表面的微观结构, 包括肉眼看不见的 病损边界和结构。近年来, 医用放大镜主要应用 于牙体牙髓病科、口腔修复科和口腔领面外科等 口腔临床科室 ${ }^{[3-5]}$, 其对于根管治疗、备牙、根尖手 术等操作具有重要的辅助价值 ${ }^{[6-8]}$ 。本研究通过 临床诊断性试验研究及临床问卷调查, 评估医用 放大镜在提高口腔黏膜病临床诊断效率中的作 用,并探讨最适合的临床参数。

\section{1 资料与方法}

\section{1 仪器}

2.5 倍眼镜式放大镜、3.5 倍头戴式放大镜和 5 倍头戴式放大镜均为德国 Carl Zeiss 公司产品, 放 大镜的具体参数见表 1 。

\section{2 病例资料}

收集 2018 年 10 月 1 日至 2019 年 4 月 30 日于

表 1 医用放大镜参数

Table 1 Parameters of medical magnifying loupes

\begin{tabular}{lccc}
\hline \multicolumn{1}{c}{ 放大镜类型 } & 放大倍数 & $\begin{array}{c}\text { 工作距离 } \\
(\mathrm{mm})\end{array}$ & $\begin{array}{c}\text { 视场直径 } \\
(\mathrm{mm})\end{array}$ \\
\hline 2.5倍眼镜式放大镜 & 2.5 & 350 & 77 \\
3.5倍头戴式放大镜 & 3.5 & 400 & 86 \\
5倍头戴式放大镜 & 5.0 & 300 & 44 \\
\hline
\end{tabular}


四川大学华西口腔医院口腔黏膜病科就诊的口腔 扁平苔藓(19例)和口腔白斑病 (5 例)患者的临床 资料用于放大镜诊断效率的评价。纳人标准: 采 用传统肉眼视诊或结合医用放大镜进行辅助视诊 诊断为斑块型口腔扁平苔藓或均质型口腔白斑 病, 最终经组织病理学活检诊断为口腔扁平苔藓 或口腔白斑病, 且对本研究知情同意并配合者。 排除标准:合并严重全身系统性疾病、恶性肿瘤及 精神疾病者; 病史资料不全患者。24例患者中男 性 8 例, 女性 16 例, 年龄 26 68 岁, 平均年龄 $(51 \pm$ 13)岁。

收集 2019年7月 1 日至2019年7月 15 日于四 川大学华西口腔医院黏膜病科就诊的各类口腔黏 膜病患者共 182 例用于放大镜使用体验的主观有 效率评价, 其中临床诊断为口腔扁平苔藓 68 例, 灼 口综合征 37 例, 复发性阿弗他溃疡 20 例, 口腔白 斑病 15 例, 其他口腔黏膜病 42 例 ; 33 例 ( $18 \%$ )通 过2.5 倍眼镜式放大镜诊断, 103 例 (57\%)通过 3.5 倍头戴式放大镜诊断,46例 (25\%)通过 5 倍头戴式 放大镜诊断。所有患者均根据相应的病史、临床 表现和病理检查结果综合作出诊断。

本研究经四川大学华西口腔医院伦理委员会 批准(WCHSIRB-D-2021-185)。

\section{3 医用放大镜佩戴及检查方法}

眼镜式放大镜佩戴方法: 首先将放大镜佩戴 于鼻梁上, 收紧环绕头部的松紧带以固定放大镜; 然后调节放大镜顶部的滑轨使镜头与医生眼部距 离合适; 最后将测试图置于放大镜工作距离处, 清 晰聚焦后通过双侧旋钮调节放大镜曈距, 使两眼 的图像重合获得均匀的圆形图像。

头戴式放大镜佩戴方法: 选择合适型号的医 用头戴式放大镜, 首先将其戴于头部, 通过后部旋 钮及顶部头带调节头部周长以固定放大镜; 然后 通过前部旋钮调节放大镜支架部分, 以使其与医 生眼部高度及距离合适; 最后将测试图置于放大 镜工作距离处, 清晰聚焦后调节放大镜瞳距, 使两 眼的图像重合获得均匀的圆形图像并固定调 节杆。

检查方法: 清洁口腔黏膜待查部位, 去除病损 部位多余唾液,避免过度反光影响观察; 在医用放 大镜下观察并记录病损区域内黏膜的色泽与质地 改变, 病损表面细微结构的特点以及病损的边界 信息等。

\section{4 医用放大镜的辅助诊断效率评估}

针对斑块型口腔扁平苔藓和均质型口腔白斑 病, 以肉眼或医用放大镜辅助肉眼分别视诊, 以组 织病理学检查结果为金标准,计算两种视诊方法 在诊断两种疾病中的敏感度、特异度、准确度、漏 诊率和误诊率, 从而判断医用放大镜的辅助诊断 效率。

\section{5 医用放大镜的使用体验评价}

设计主观有效率调查问卷, 记录并统计临床 医生使用医用放大镜辅助视诊不同口腔黏膜病效 率的主观评价,包括口腔黏膜病种类、放大镜倍数 及放大镜辅助是否有效。

设计使用体验调查问卷,记录三种不同型号 放大镜的使用体验和不良感受等,包括成像清晰 度、放大倍数、视野大小、工作质量、工作距离、长 时间佩戴舒适度、视疲劳和眩晕感等。问卷分别 由 31 位口腔黏膜病科专科医生和从事口腔黏膜临 床工作 1 年以上的口腔黏膜病学专业研究生独立 完成。

\section{6 统计学方法}

采用SPSS 10.0 软件进行统计分析,计数资料 采用 $\chi^{2}$ 检验或 Fisher 确切概率法, $P<0.05$ 为差异有 统计学意义。

\section{2 结 果}

\section{1 医用放大镜的辅助诊断效率}

24 例患者肉眼视诊和放大镜检查结果见 表 2 。医用放大镜辅助视诊鉴别斑块型口腔扁平 苔藓和均质型口腔白斑病的诊断敏感度、特异度、 准确度分别为 $94.74 \% 、 100.00 \% 、 95.83 \%$,均高 于肉眼视诊 $(89.47 \% 、 80.00 \% 、 87.50 \%)$; 医用 放大镜辅助视诊的漏诊率、误诊率分别为 $5.26 \%$ 、

表 2 肉眼视诊和使用医用放大镜辅助视诊鉴别口腔扁 平苔藓和口腔白斑病结果

Table 2 Results of naked eye inspection and medical magnifying loupes to distinguish oral lichen planus and oral leukoplakia

$(n)$

\begin{tabular}{|c|c|c|c|c|c|}
\hline \multirow[b]{2}{*}{ 病理检查结果 } & \multirow[b]{2}{*}{$n$} & \multicolumn{2}{|c|}{ 肉眼视诊 } & \multicolumn{2}{|c|}{ 放大镜辅助诊断 } \\
\hline & & $\begin{array}{l}\text { 口腔扁 } \\
\text { 平苔藓 }\end{array}$ & $\begin{array}{c}\text { 口腔白 } \\
\text { 斑病 }\end{array}$ & $\begin{array}{c}\text { 口腔扁平 } \\
\text { 苔藓 }\end{array}$ & $\begin{array}{l}\text { 口腔白 } \\
\text { 斑病 }\end{array}$ \\
\hline 口腔扁平苔蘚 & 19 & 17 & 2 & 18 & 1 \\
\hline 口腔白斑病 & 5 & 1 & 4 & 0 & 5 \\
\hline 合 计 & 24 & 18 & 6 & 18 & 6 \\
\hline
\end{tabular}


$0.00 \%$, 均低于肉眼视诊 $(10.53 \%, 20.00 \%)$ 。以 下介绍 4 例较为典型的病例诊断。

病例 1 : 患者右舌腹处有一直径约为 $2 \mathrm{~mm}$ 的 白色斑块伴大小约 $20 \mathrm{~mm} \times 10 \mathrm{~mm}$ 的白色斑片, 病损 前份边界清晰,角化程度较高; 病损后份边界不清, 角化程度较低。肉眼视诊诊断: 疑似口腔白斑病、 疑似斑块型口腔扁平苔藓。医用放大镜辅助视诊 见大小为 $30 \mathrm{~mm} \times 15 \mathrm{~mm}$ 的白色斑片, 病损与正常 黏膜边界清晰 (图 1), 诊断为口腔白斑病。根据病 理检查结果, 该例患者确诊为口腔白斑病。

病例 2 : 患者舌背处有 3 个直径约为 $6 \mathrm{~mm}$ 的白 色斑块, 肉眼视诊诊断: 疑似斑块型口腔扁平苔 藓、疑似口腔白斑病。医用放大镜辅助视诊见舌 背病损区域部分丝状乳头萎缩, 表面可见网状白 纹,白色斑块病损边界不清,边缘呈放射状(图2), 诊断为斑块型口腔扁平苔藓。根据病理检查结 果,该例患者确诊为口腔扁平苔藓。

病例 3 : 患者左颊黏膜中后份有一网状白纹, 可见两处局部充血面,其下份充血面中央见一直径

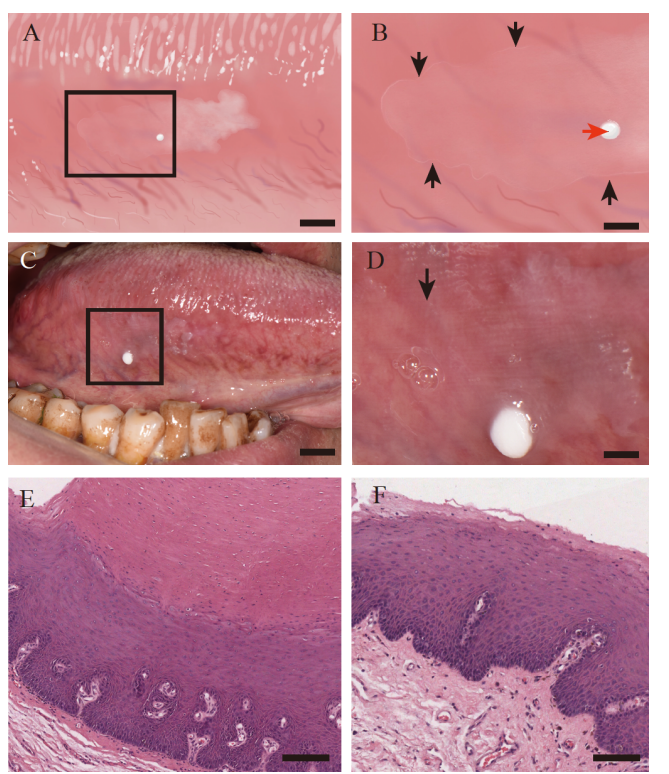

$\mathrm{A} 、 \mathrm{~B}$ : 病理模式图 (图B为图 A中黑色方框区域放大图片), 可 见医用放大镜辅助视诊能放大病损边缘微观结构 (红色箭头指示 高角化病损, 黑色箭头指示病损后份边界), 有利于确定病损与正 常黏膜边界; C、D : 口腔内放大后的病损局部照片 (图D为图C中黑 色方框区域放大图片), 可观察到病损后份与正常黏膜边界清晰 (箭头所示), 符合医用放大镜辅助视诊结果; $\mathrm{E} 、 \mathrm{~F}: \mathrm{HE}$ 染色图, 可见 病损前份及后份均有上皮增生伴过度不全角化, 粒层明显, 棘层增 厚, 符合口腔白斑病的诊断. 图 $\mathrm{A} 、 \mathrm{C}$ 标尺 $=5 \mathrm{~mm}$, 图 $\mathrm{B} 、 \mathrm{D}$ 标尺 $=2 \mathrm{~mm}$, 图 $\mathrm{E}$ 标尺 $=100 \mu \mathrm{m}$, 图F标尺 $=50 \mu \mathrm{m}$.

图 1 口腔白斑病(病例1)病损图

Figure 1 Images of the lesions in patient with oral leukoplakia (case 1)
约 $5 \mathrm{~mm}$ 的浅糜烂, 肉眼视诊诊断: 糜烂型口腔扁平 苔藓。医用放大镜辅助视诊观察到左项黏膜上份 充血面散在肉眼视诊不可见的数个针尖大小糜烂 面 (图 3), 诊断为糜烂型口腔扁平苔藓。根据病理 检查结果, 该例患者确诊为口腔扁平苔藓。

病例 4 : 患者全口牙龈有大小不等的散在糜 烂面, 肉眼视诊诊断: 疑似大疮性疾病、疑似复发 性阿弗他溃疡。医用放大镜辅助视诊见牙龈糜烂 面周围有残余疮壁 (图 4), 修改诊断为寻常型天疮 疮。根据病理检查结果, 该例患者确诊为天 疮疮。

综上, 医用放大镜通过辅助对肉眼不可见病 损的识别可提高视诊的临床诊断效率, 降低漏诊 率和误诊率。

\section{2 医用放大镜使用体验调查结果}

2.2.1 主观有效率评价调查结果显示, 医用放 大镜辅助诊断的总有效率为 $91.76 \%(167 / 182)$ 。

其中, 33 例通过 2.5 倍眼镜式放大镜辅助诊断的 病例中, 报告其有助于诊断 26 例, 有效率为 $78.79 \% ; 103$ 例通过 3.5 倍头戴式放大镜辅助诊断 的病例中, 报告其有助于诊断 97 例, 有效率为 $94.17 \%$; 46 例通过 5 倍头戴式放大镜辅助诊断的 病例中, 报告其有助于诊断 44 例, 有效率为 $95.65 \%$ 。3. 5 倍头戴式放大镜和 5 倍头戴式放大 镜辅助诊断的主观有效率均高于 2.5 倍眼镜式放 大镜 $($ 均 $P<0.05)$, 而 3.5 倍头戴式放大镜和 5 倍头 戴式放大镜之间主观有效率差异无统计学意义 $(P>0.05)$ 。为避免因样本数过少引起的误差, 选 择常见口腔黏膜病 (至少 15 例患者)进行分析, 包 括口腔扁平苔藓(68例)、口腔白斑病 (15 例)、灼口 综合征(37例)、复发性阿弗他溃疡(20例)。结果 显示, 医用放大镜在各类口腔黏膜病诊断中的主 观有效率分别为口腔白斑病 $100.00 \%$ 、口腔扁平 苔藓 $98.53 \%$ 、灼口综合征 $83.78 \%$ 、复发性阿弗他 溃疡 75\%, 见图 5。结果提示, 医用放大镜对包括 口腔扁平苔藓、灼口综合征、口腔白斑病、复发性 阿弗他溃疡在内的口腔黏膜病的视诊均具有一定 的辅助作用。

2.2.2 最适放大倍数和最适工作距离 调查 结果显示, 在“成像清晰”、“放大倍数合 适”、“视野大小合适” 和 “工作质量提升” 这 四项中, 3.5 倍头戴式放大镜的使用满意度最高, 与另外两种放大镜差异有统计学意义 (均 

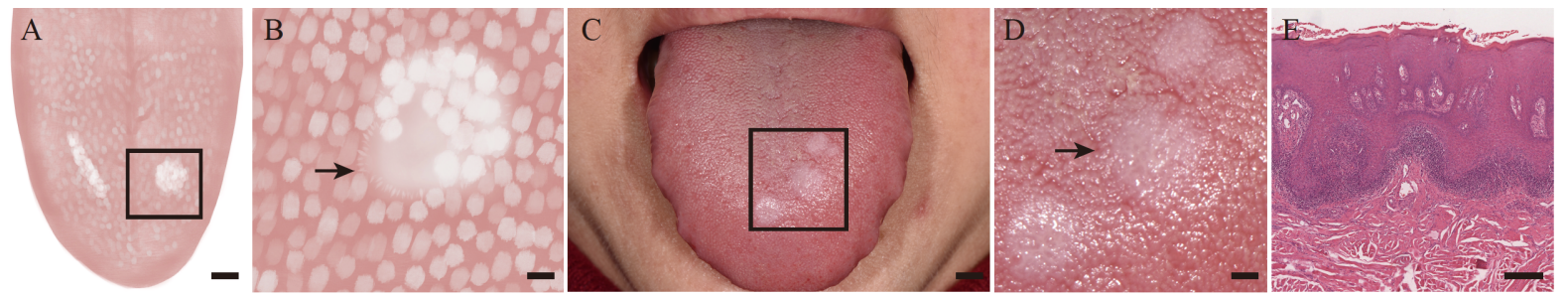

$A 、 B$ : 病理模式图 (图B为图A中黑色方框区域放大图片),显示医用放大镜辅助视诊可放大病损边缘形态,镜下可清晰观察到病损边缘 放射状白纹 (箭头所示); C、D : 口腔内放大后的病损局部照片 (图D为图C中黑色方框区域放大图片), 可观察到其边界不清,边缘呈放射状白 纹(箭头所示), 符合医用放大镜辅助视诊结果; $\mathrm{E}: \mathrm{HE}$ 染色图,可见病损上皮钉突不规则伸长,基底细胞液化变性,固有层淋巴细胞带状浸 润,符合口腔扁平苔藓的诊断. 图 $\mathrm{A} 、 \mathrm{C}$ 标尺 $=8 \mathrm{~mm}$, 图B、D标尺 $=2 \mathrm{~mm}$,图E标尺 $=150 \mu \mathrm{m}$.

图 2 斑块型口腔扁平苔藓(病例2)病损图

Figure 2 Images of the lesions in patient with plaque-type oral lichen planus (case 2)
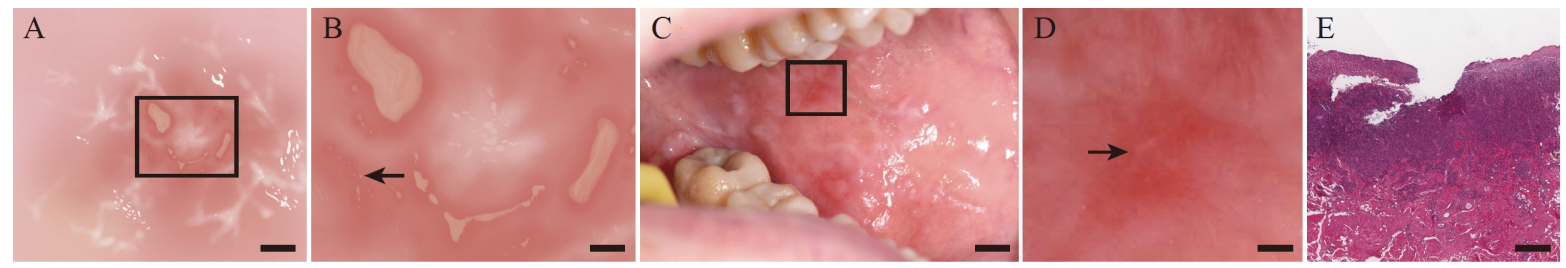

$A 、 B$ : 病理模式图(图B为图A中黑色方框区域放大图片), 显示医用放大镜辅助视诊可放大病损微观结构,利于确定病损范围,在镜下 可清晰观察到病损的微小糜烂面 (箭头所示);C、D : 口腔内放大后的病损局部照片 (图D为图C中黑色方框区域放大图片), 可观察到充血区 域表面的微小糜烂面 (箭头所示),符合医用放大镜辅助视诊结果; $\mathrm{E}: \mathrm{HE}$ 染色图,可见病损上皮缺损,基底细胞液化变性,固有层淋巴细胞带 状浸润,符合口腔扁平苔藓的诊断. 图 $\mathrm{A} 、 \mathrm{C}$ 标尺 $=5 \mathrm{~mm}$, 图B、D标尺 $=1 \mathrm{~mm}$, 图 $\mathrm{E}$ 标尺 $=150 \mu \mathrm{m}$.

图 3 糜烂型口腔扁平苔藓(病例3)病损图

Figure 3 Images of the lesions in patient with erosive oral lichen planus (case 3)
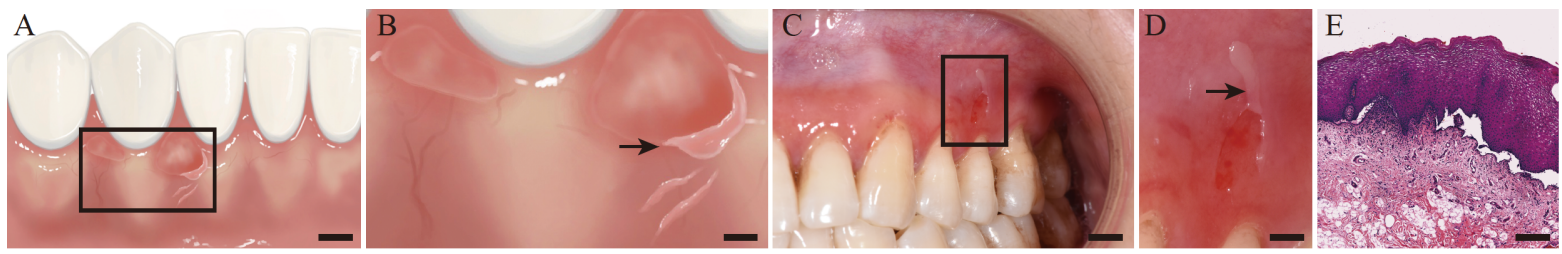

$A 、 B$ : 病理模式图 (图B 为图A中黑色方框区域放大图片), 显示医用放大镜辅助视诊可放大病损隐匿结构,在镜下可清晰观察到病损糜 烂面周围残余疮壁(箭头所示); C、D : 口内照片 (图D为图C中黑色方框区域放大图片),放大后的病损局部照片可观察到糜烂面周围有残余 疮壁 (箭头所示), 符合医用放大镜辅助视诊结果; $\mathrm{E}: \mathrm{HE}$ 染色图, 可见病损棘层松解, 上皮内疮形成,符合天疮疮的诊断. 图 $\mathrm{A} 、 \mathrm{C}$ 标尺 $=5 \mathrm{~mm}$, 图B标尺 $=2 \mathrm{~mm}$, 图D标尺 $=1.25 \mathrm{~mm}$, 图E标尺 $=100 \mu \mathrm{m}$.

图 4 寻常型天疮疮(病例4)病损图

Figure 4 Images of the lesions in patient with pemphigus vulgaris (case 4)

$P<0.05)$; 在 “工作距离合适” 的评价中, 3.5 倍头 戴式放大镜 $(400 \mathrm{~mm})$ 和 2.5 倍眼镜式放大镜 $(350 \mathrm{~mm})$ 结果相似,均高于 5 倍头戴式放大镜 $(300 \mathrm{~mm}$, 均 $P<0.05)$, 见图 6。结果提示, 大部分 医生认为 3.5 倍头戴式放大镜的成像清晰度、放大 倍数、视野大小、工作质量提升度以及合适的工作 距离最佳。

2.2.3 佩戴者不良感受评估 在 “长时间佩戴 不适感” 的评价中, 3.5 倍头戴式放大镜和 2.5 倍 眼镜式放大镜结果相似, 且均明显低于 5 倍头戴式 放大镜 $($ 均 $P<0.05)$; 在 “视觉疲劳” 和 “眩晕
感” 的两种评价中, 3.5 倍头戴式放大镜评价居 中, 但低于 5 倍头戴式放大镜的上述两项不良感受 $($ 均 $P<0.05)$, 见图 6。结果提示, 大部分临床医生 认为 5 倍头戴式放大镜具有明显的长时间佩戴不 适感、视觉疲劳和眩晕感。

\section{3 讨 论}

病损的重叠性是口腔黏膜病的重要临床特 点。受人眼视敏度等生理因素的制约,传统肉眼 视诊检查的准确性受到影响 ${ }^{[9]}$ 。人眼识别物体的 敏感性通常通过视敏度来表示, 视敏度表示人眼 


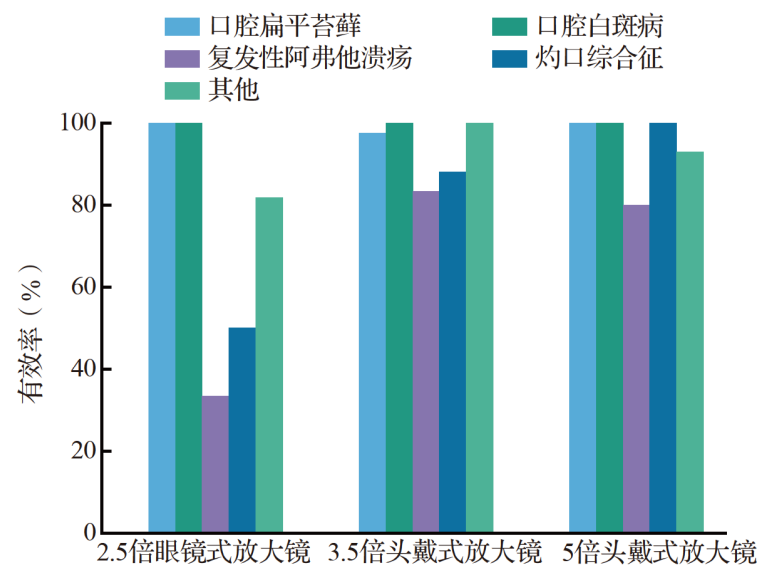

图 5 不同医用放大镜在各种口腔黏膜疾病诊断中的主 观有效率

Figure 5 The subjective effectiveness of different medical magnifying loupes in diagnosis

在一定距离 $(l)$ 内辨别相隔最近 $(d)$ 的两个点的 能力。视敏角为两个点和眼睛之间形成的角度 $\theta$ (图7), 视敏角越小, 视敏度越高, 反之亦然。正常 人眼可以达到的最小视敏角为 $1^{\circ[10]}$ 。

目前, 口腔黏膜病科临床诊断缺乏简单、快 速、有效的辅助诊断工具。医用放大镜是一种非 侵人性的视觉放大技术, 通过增加人肉眼视敏 度 ${ }^{[11]}$, 可用于观察口腔黏膜表面的微观结构,包 括肉眼看不见的病损边界结构。医用放大镜具有 无创、方便、实时、成像清晰等特点, 符合口腔黏膜 病临床辅助诊断的需求。

在视诊中, 临床医生通常通过肉眼视诊观察 病损并根据其临床特征进行诊断, 但很可能会错
过肉眼不可见的微小病损。医用放大镜可以将微 小病损和结构放大至 2.5 5倍, 有助于医生识别肉 眼不可见的病损,从而提高诊断效率。本研究评 估了医用放大镜在口腔黏膜病辅助诊断中的效 率, 结果可见医用放大镜提高了人眼视敏度, 辅助 常规肉眼视诊能获得更高的敏感度和特异度, 从 而提升肉眼视诊对微小、隐匿病损的信息捕获和 判断, 降低漏诊率和误诊率。在对辅助诊断效率 的主观评价中, 临床医生认为医用放大镜对口腔 扁平苔藓、灼口综合征、口腔白斑病、复发性阿弗 他溃疡及其他口腔黏膜病的检查均有辅助作用。

口腔科医生佩戴医用放大镜也存在长时间佩 戴不适感、视觉疲劳、眩晕感等不良反应 ${ }^{[12-14]}$ 。在 最适合的临床使用参数研究中, 本文资料显示长 时间佩戴不适感、视觉疲劳和眩晕感等不良反应 或感受与医用放大镜的放大倍率呈正相关, 放大 倍数越高, 产生负面感受的可能性就越大。这也 与视野大小存在一定关系, 放大倍数越高, 视野就 越小, 在视野中观察到的病损范围就越有限, 这就 需要连续移动放大镜, 增加了移动过程中眩晕的 可能性。在工作距离的调查中, 本研究发现大多 数医生认为小于或等于 $300 \mathrm{~mm}$ 的工作距离因与患 者的距离太近会增加接触感染的风险,推荐采用 $400 \mathrm{~mm}$ 的工作距离。

综上, 医用放大镜具有成像清晰、实时、方便、 无创等特点, 能有效提高口腔黏膜病的临床诊断 效率, 是值得推荐的辅助诊断工具。综合辅助诊

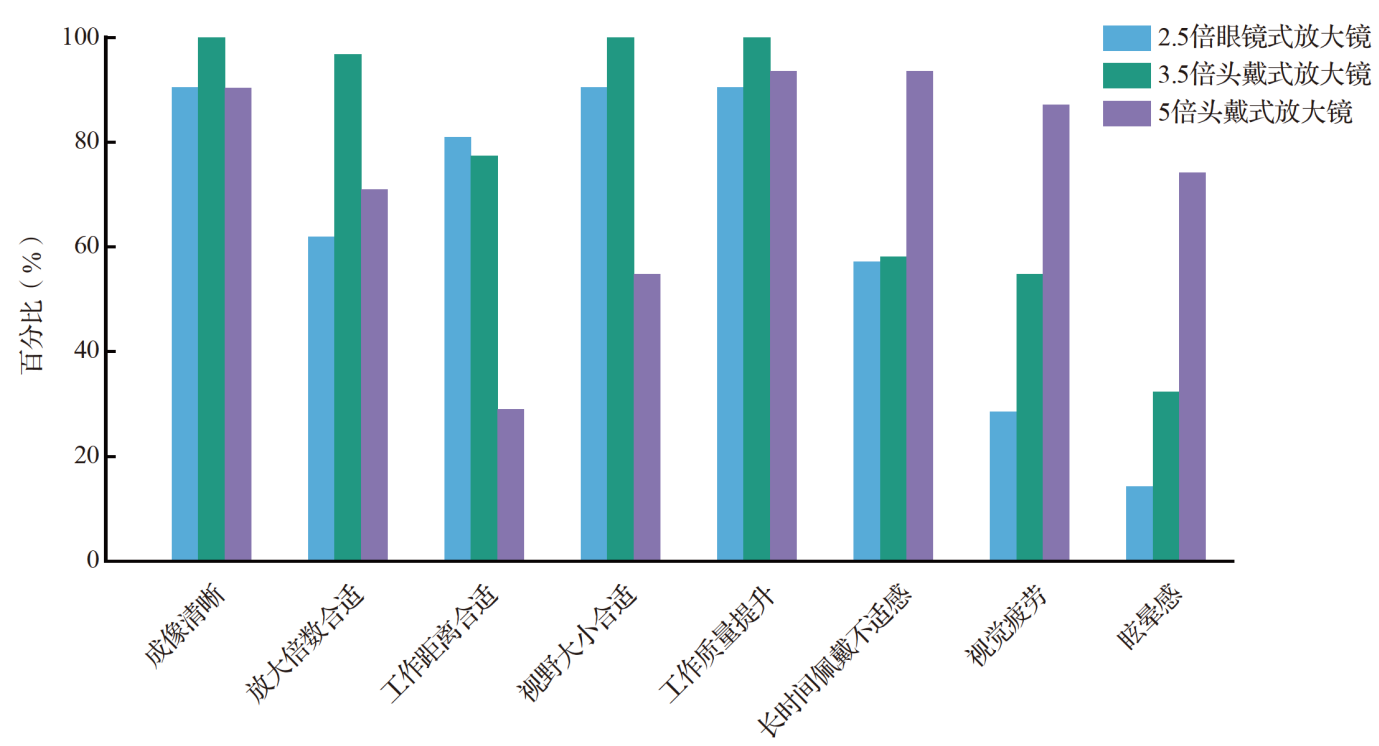

图 6 医生对不同医用放大镜的使用体验分布

Figure 6 Physician's experience of using different medical magnifying loupes 


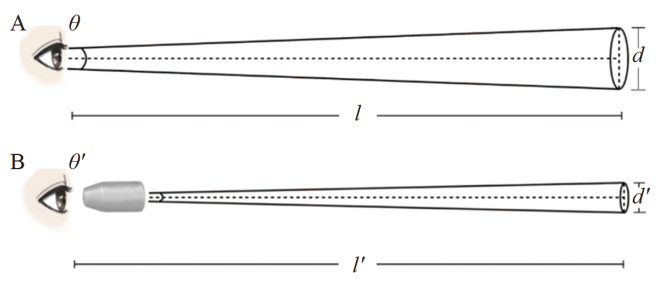

$\mathrm{A}$ : 裸眼视物; $\mathrm{B}$ : 医用放大镜辅助视物. $l$ : 视物距离; $d$ : 裸眼辨 别两点间最小间距; $\theta$ : 裸眼视敏角; $l^{\prime}$ : 放大镜视物距离; $d^{\prime}$ : 放大镜 辨别两点间最小间距; $\theta^{\prime}$ : 放大镜视敏角.

图 7 裸眼视敏角和应用医用放大镜减小视敏角示意图

Figure 7 Schematic diagrams of naked eye visual acuity angle and the application of medical magnifying loupes to reduce visual acuity angle

断效能和使用舒适度, 推荐口腔黏膜病临床诊疗 使用 3.5 倍放大倍数, 工作距离 $400 \mathrm{~mm}$ 的医用放 大镜。

\section{利益冲突 所有作者均声明不存在利益冲突}

\section{参考文献}

[1] 陈谦明. 口腔黏膜病学 [M]. 北京: 人民卫生出版社, 2012: 1-2.

CHEN Qianming. Oral Mucosal Diseases $[\mathrm{M}]$. Beijing: People's Medical Publishing House, 2012: 12. (in Chinese)

[2] BAKER J M, MEALS R A. A practical guide to surgical loupes[J]. J Hand Surg Am, 1997, 22(6): 967-974.

[3] FRIEDMAN M J. Magnification in a restorative dental practice: from loupes to microscopes $[\mathrm{J}]$. Compend Contin Educ Dent, 2004, 25(1): 48, 50, 53-55.

[4] PERRIN P, NEUHAUS K W, LUSSI A. The impact of loupes and microscopes on vision in endodontics $[\mathrm{J}]$. Int Endod J, 2014, 47(5): 425-429.

[5] 苗 磊, 潘亚萍. 口腔显微技术发展与应用 $[\mathrm{J}]$. 中国 实用口腔科杂志, 2014, 7(4): 196-199.

MIAO Lei, PAN Yaping. Development and application of microscopy in oral treatment $[\mathrm{J}]$. Chinese Journal of Practical Stomatology, 2014, 7(4): 196-199. (in Chinese)
[6] HASAN M, KHAN F R. Determination of frequency of the second mesiobuccal canal in the permanent maxillary first molar teeth with magnification loupes (×3.5)[J]. Int J Biomed Sci, 2014, 10(3): 201-207.

[7] TASCHIERI S, WEINSTEIN T, TSESIS I, et al. Magnifying loupes versus surgical microscope in endodontic surgery: a four-year retrospective study $[\mathrm{J}]$. Aust Endod J, 2013, 39(2): 78-80.

[8] 葛严军, 刘晓强. 放大镜与显微镜辅助下瓷贴面牙体 预备效果的比较 $[\mathrm{J}]$. 北京大学学报(医学版)，2019, 51(1): 100-104.

GE Yanjun, LIU Xiaoqiang. Effects of loupes and microscope on laminate veneer preparation $[\mathrm{J}]$. Journal of Peking University (Health Sciences), 2019, 51(1): 100-104. (in Chinese)

[9] EICHENBERGER M, PERRIN P, NEUHAUS K W, et al. Visual acuity of dentists under simulated clinical conditions $[J]$. Clin Oral Invest, 2013, 17(3): 725729.

[10 ] 章 萍. 人眼的视觉敏锐度 $[\mathrm{J}]$. 河南医科大学学报, 1987(1): 30-32.

ZHANG Ping. Visual acuity of human eyes[J]. Journal of Henan Medical University, 1987(1): 30-32. (in Chinese)

[11 ] PERRIN P, EICHENBERGER M, NEUHAUS K W, et al. Visual acuity and magnification devices in dentistry [J]. Swiss Dent J, 2016, 126(3): 222-235.

[12] JAMES T, GILMOUR A S M. Magnifying loupes in modern dental practice: an update $[\mathrm{J}]$. Dent Update, 2010, 37(9): 633-636.

[13 ] HAYES M J, TAYLOR J A, SMITH D R. Introducing loupes to clinical practice: dental hygienists experiences and opinions [J]. Int J Dent Hyg, 2016, 14(3): 226-230.

[14] EICHENBERGER M, PERRIN P, RAMSEYER S T, et al. Visual acuity and experience with magnification devices in Swiss dental practices $[\mathrm{J} / \mathrm{OL}]$. Oper Dent, 2015, 40(4): E142-E149.

$$
\text { [本文编辑 沈 敏 余 方] }
$$

